\section{EXTREMELY STRONG, STABLE DENTAL CHAIRS}

According to recent statistics $63.8 \%$ of adults in England have a BMI or 25 and over. It is reported that half of UK males could be obese by 2030 if current trends continue. Hopefully that projection will not ever come to fruition; however, there will always be a proportion of the population who are severely overweight.

Planning for the future has always been at the forefront of Takara Belmont's R\&D programme and the company is pleased to report that the weight lifting capacity of Takara Belmont's chairs has increased to 22 stones when a spittoon or over the patient unit is attached to the chair. For standalone chairs, includ- ing the Clesta I \& II, 037 Pro II and Clair chairs, the limit has increased to 31 stone.

Takara Belmont chairs are therefore extremely strong and stable, so will meet the needs of certain bariatric patients without compromising aesthetics or comfort. For further information or clarification contact a member of the Technical Support Department on 020 75150333 or email dental@takara. co.uk.

\section{UNIVERSITIES JOIN ORTHODONTIC TRAINING PROGRAMME}

Align Technology has reached agreement with a further four dental schools to join its orthodontic training programme, bringing the number at which it teaches to seven. The Invisalign University Programme has been deemed an integral part of the course for postgraduate dentists as they train to be orthodontists, which recognises that aligner treatments are now accepted as a mainstream orthodontic treatment.

Since its inception in 2013 the programme has been run at Queen Mary's Hospital in Sidcup, Kent, the Countess of Chester Hospital and Dublin Dental Hospital. These early adopters realised the advantage to train their orthodontic students in aligner techniques before they qualify as Specialists to ensure graduates are au fait with the latest techniques they are likely to be asked about in practice. The expanded programme is now also to be offered at the universities of Leeds, Cardiff, Newcastle and Sheffield.

Each university programme gives up to 20 orthodontic students in every intake the opportunity to undergo training from experienced orthodontic specialists who are skilled in the use of the Invisalign orthodontic appliance and can share both theoretical and practical expertise. Tutors include Dr Graham Gardner who has completed over 650 Invisalign cases and sessions are complemented by practical sessions from Align's Clinical Advisors.

The Invisalign University Training Programme starts with an introduction to the Invisalign Doctor Site; treatment options and treatment selection; record submission; the use of the ClinCheck software and aligner delivery; and monitoring and finishing. Each university is provided with Invisalign materials and access to the Clincheck software programme.

An authorised member of the faculty of universities interested in adding Invisalign to its orthodontic training curriculum is requested to email: eu-universities@aligntech for more information.

\section{THE ONLY DUAL-CURING UNIVERSAL ADHESIVE}

VOCO presents several innovative products at the British Dental Conference and Exhibition 2014. One is Futurabond U, the market's only true dual-curing universal adhesive offering usage in a disposable Single-

Dose applicator.

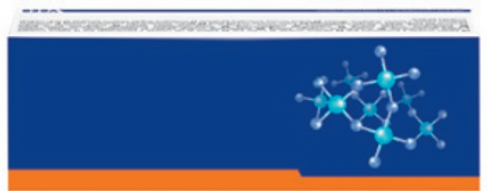

Futurabond U

offers an out-

standing range of options for application, as much with regard to indica- tions as to selection of the etching technique or the curing mode. Applied in a single layer, this new universal adhesive creates a strong bond to enamel and dentine, thus ensuring a durable, gap-free bond between the dental hard tissue and the restorative material. Futurabond $\mathrm{U}$ is fully compatible with all light-curing, dual-curing and self-curing methacrylate-based composites. At the same time it offers firm adhesion to different materials such as metal, zirco-

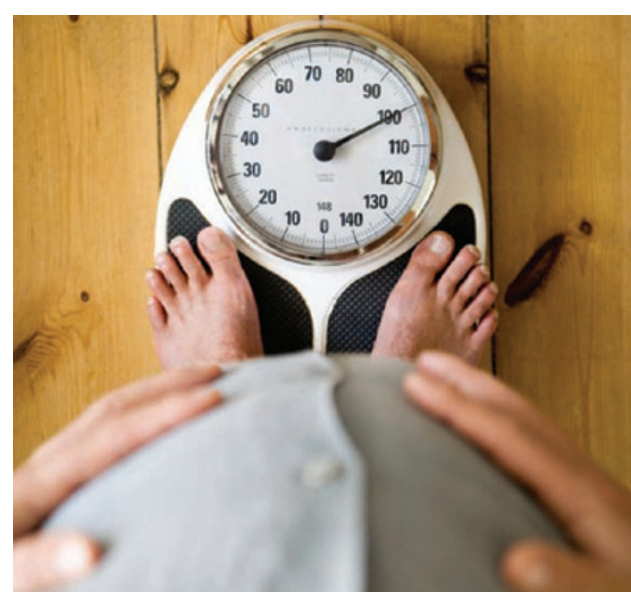

TAKE YOUR DENTISTRY TO THE NEXT LEVEL

The British Academy of Cosmetic Dentistry (BACD) will have a strong presence at this year's British Dental Conference and Exhibition.

By visiting stand D47 you can meet like-minded colleagues who all share the same passion to simply 'get better at what they do'. You will also be able to find out more about the BACD's range of activities, including Regional Meetings, lecture days and the Annual Conference in November.

The BACD will also be hosting a range of lectures as part of the 'Accessible Cosmetic Dentistry' programme.

Make sure you visit the BACD at stand D47, or attend a BACD lecture. www.bacd.com nium/aluminium oxide and silicate ceramics without any additional primer. The patented SingleDose guarantees fast, simple working as mixing errors are ruled out from the start, and the product is very hygienic. The new bonding material comes in SingleDose packs of 50 and 200.

Visit www.voco.com or email info-uk@voco.com. VOC0 will be on stand A37 at the British Dental Conference \&t Exhibition 2014 in Manchester. 